\title{
Nitro complexos de ferro(III) com derivados da base de Schiff salen
}

Anallicy Santos de Paiva* (Doutoranda do PPGQ na Universidade Federal do RN- UFRN) Francisco Ordelei Nascimento da Silva (Prof. Adj. do IQ na Universidade Federal do RN- UFRN) Ana Cristina Facundo de Brito Pontes (Profa. Adj. do IQ na Universidade Federal do RN - UFRN)

Daniel de Lima Pontes (Prof. Adj. do IQ na Universidade Federal do RN - UFRN)

* Email: anallicy@hotmail.com

Resumo: Os complexos com a base de Schiff salen apresentam relevantes aplicações como agentes quimioterápicos e na química de materiais. Buscando desenvolver compostos de coordenação que intensifiquem as propriedades farmacológicas dos complexos contendo bases de Schiff, utilizou-se o íon nitrito para acentuar a atividade biológica devido as características dos nitro complexos de atuarem como pró-droga para a liberação de óxido nítrico em meio biológico, um importante agente de proteção celular e vasodilatador. No presente trabalho foram obtidos novos nitro complexos de ferro(III) com bases de Schiff derivados do salen, sendo sintetizados os ligantes 3-MeOsalen e o 3-MeOsalophen a partir das diaminas etilenodiamina e orto-fenilenodiamina, respectivamente e o aldeído a partir da orto-vanilina em ambos os ligantes. Os compostos 3-MeOsalen e 3-MeOsalophen e os complexos [Fe(3-MeOsalen)Cl], [Fe(3-MeOsalophen)Cl], [Fe(3-MeOsalen) $\mathrm{NO}_{2}$ ] e [Fe(3-MeOsalophen) $\mathrm{NO}_{2}$ ] foram caracterizados através da espectroscopia vibracional na região do infravermelho e a espectroscopia eletrônica na região do ultravioleta e visível.

A partir da análise dos espectros de infravermelho, foi possível caracterizar os ligantes 3-MeOsalen e 3-MeOsalophen, principalmente, pela presença da banda referente ao $v \mathrm{C}=\mathrm{N}$ próximo a $1620 \mathrm{~cm}^{-1}$. A formação dos complexos precursores foi evidenciada pelas alterações nas frequências de vibração $v \mathrm{C}=\mathrm{N}$ e $v \mathrm{C}-\mathrm{O}$ e pelo surgimento de modos vibracionais metal-oxigênio e metal-nitrogênio. Para os nitro complexos de ferro foram exibidos os $v\left(\mathrm{NO}_{2}\right)_{\text {ass }}$ em torno de $1300 \mathrm{~cm}^{-1}$ e $v\left(\mathrm{NO}_{2}\right)_{\operatorname{sim}}$ em $1271 \mathrm{~cm}^{-1}$, além da deformação angular em torno de $830 \mathrm{~cm}^{-1}$, indicando que a coordenação do íon nitrito ocorreu via átomo de nitrogênio.

Os espectros eletrônicos das bases de Schiff são marcadas pela presença das transições $\pi-\pi^{*}$ do anel aromático e do grupo imino, além da banda $n-\pi^{*}$ referente as transições dos elétrons não ligantes. Os espectros dos complexos precursores [Fe(3-MeOsalen)Cl] e [Fe(3MeOsalophen)Cl] apresentaram deslocamentos das bandas de transições intraligantes e ainda novas bandas referentes a LMCT $\left(\mathrm{p} \pi \mathrm{Cl}^{-} \rightarrow \mathrm{d} \pi^{*} \mathrm{Fe}^{3+}\right)$. Já nos espectros dos compostos [Fe(3-MeOsalen) $\mathrm{NO}_{2}$ ] e [Fe(3-MeOsalophen) $\mathrm{NO}_{2}$ ] destacaram-se a ausência da banda LMCT presente no complexo precursor.

Palavras-chave: Complexos; base de schiff; salen; nitro. 


\section{I ntrodução}

O desenvolvimento de novos compostos de coordenação com aplicação na área biológica tem se intensificado nos últimos anos (MUHAMMAD; GUO, 2014). Estudos das propriedades terapêuticas e farmacológicas dos complexos, bem como a investigação e a compreensão dos mecanismos de interação entre estes e biomoléculas promoveram avanços relevantes na área da química bioinorgânica.

Entre os ligantes constituintes dos compostos de coordenação, as bases de Schiff têm aplicações bastante amplas e de grande importância, por originar complexos estáveis com diversos metais em estados de oxidação variados (COZZI, 2004).

$\mathrm{O}$ principal ligante polidentado pertencente à classe das bases de Schiff é o N,N'etilenobis(salicilidenoimina), usualmente denominado de salen, o qual resulta da condensação de salicilaldeído (2-hidroxibenzaldeído) com etilenodiamina, na proporção 2 para 1 , formando um ligante tetradentado. Os complexos com ligantes salen e derivados são amplamente estudados devido as suas diferentes aplicações em catálise, atuando em reações de oxidação, redução e polimerização (GHAFFARI et al., 2014). No que se refere à atividade biológica, foi identificado que estes compostos apresentam ação citotóxica frente a tumores malignos (WOLDEMARIAM; MANDAL, 2008; LEE et al., 2011; ANSARI et al., 2009), bem como a capacidade de fragmentar o DNA (DEHKORDI; LINCOLN, 2013).

A importância da obtenção de novos nitro complexos está associado a atividade biológica presentes nestes compostos que permitem a atuação como pró-droga para liberação de óxido nítrico. Desse modo, o íon nitrito é atualmente considerado uma potencial fonte de óxido nítrico a partir de sua conversão biológica a NO em certas condições bioquímicas (ZAHRAN et al., 2008). Os compostos que funcionem como agentes liberadores de óxido nítrico têm se destacado devido esta molécula atuar como um mensageiro biológico no organismo, tendo participação no controle da pressão arterial (IGNARRO, 1989), no sistema neurotransmissor (GARTHWAITE, 1991), nas respostas imunológicas (HIBBS Jr. et al., 1988) e nas ações antioxidantes (WINK; MITCHELL, 2003).

\section{Procedimento experimental}

\subsection{Sínteses dos ligantes derivados de base de Schiff salen}

As sínteses dos ligantes foram realizadas de acordo com o procedimento similar ao descrito na literatura (ARANHA et al., 2007). As bases de Schiff foram obtidas pela reação entre um aldeído e uma diamina previamente dissolvidos em metanol, sendo utilizado a razão molar 2:1. As soluções foram mantidas sob agitação constante e sob refluxo durante duas horas. Por fim, o precipitado foi filtrado e lavado com metanol. Na preparação dos ligantes alterou-se as diaminas, sendo utilizada a etilenodiamina e a orto-fenilenodiamina e manteve-se o mesmo aldeído, a orto-vanilina, formando o 3-MeOsalen e 3-MeOsalophen com rendimento de 93\% e $95 \%$, respectivamente. Na figura 1 são apresentadas as estruturas dos ligantes.

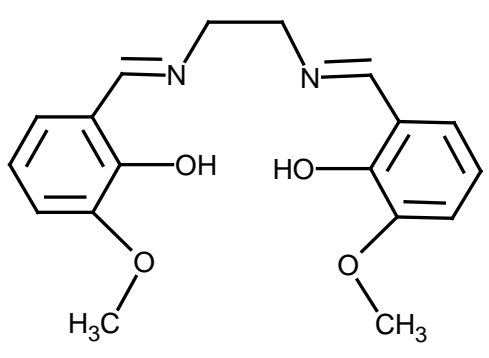

(a)

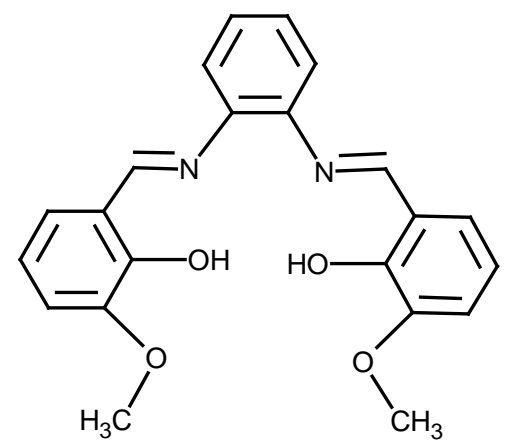

(b)

Figura 1- Estruturas dos ligantes (a) 3-MeOsalen e (b) 3-MeOsalophen 


\subsection{Sínteses dos nitro complexos com derivados da base de Schiff salen}

A partir dos ligantes apresentados, foram sintetizados dois complexos precursores de Fe(III), o [Fe(3-MeOsalen)Cl] e o [Fe(3-MeOsalophen)Cl]. Os complexos foram obtidos a partir da reação de $1 \mathrm{mmol}$ de $\mathrm{FeCl}_{3} \cdot 6 \mathrm{H}_{2} \mathrm{O}$ e $1 \mathrm{mmol}$ do ligante apropriado. As sínteses dos complexos precursores de $\mathrm{Fe}(\mathrm{III})$ foram realizadas conforme encontrado na literatura (SALOMÃO et al., 2007).

Os compostos [Fe(3-MeOsalen)Cl] e o [Fe(3-MeOsalophen)Cl] foram utilizados como compostos de partidas para a obtenção dos nitro complexos. Utilizou-se $1 \mathrm{mmol}$ do complexo de partida correspondente que foi parcialmente solubilizado em metanol. A este sistema reacional foram adicionados 2,5 mmol de nitrito de sódio previamente solubilizado em metanol. Esta solução foi mantida sob agitação e refluxo por duas horas. Depois desse período, o composto foi resfriado por 24 horas e o precipitado marrom obtido foi filtrado e lavado com metanol. O rendimento do [Fe(3-MeOsalen) $\mathrm{NO}_{2}$ ] foi de $55 \%$ e [Fe(3-MeOsalophen) $\mathrm{NO}_{2}$ ] foi de $70 \%$. As estruturas propostas dos nitro complexos são ilustradas na figura 2.

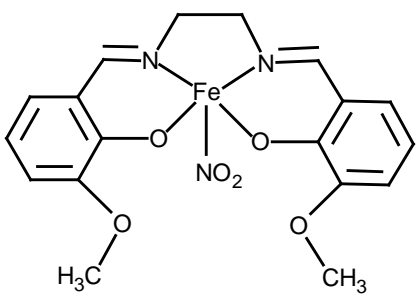

(a)

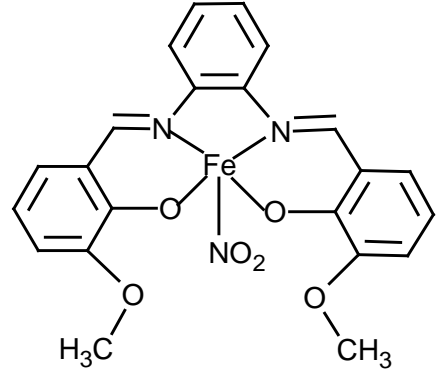

(b)

Figura 2- Estruturas propostas dos complexos (a) $\left[\mathrm{Fe}(3-\mathrm{MeOsalen}) \mathrm{NO}_{2}\right]$ e

\subsection{Métodos de caracterização}

O equipamento utilizado para obter os espectros de infravermelho foi o Espectrofotômetro Shimadzu, modelo FTIR-8400S. Todas as amostras foram preparadas em estado sólido em pastilhas de brometo de potássio $(\mathrm{KBr})$ e registradas em transmitância, na faixa do espectro de 4000 a $400 \mathrm{~cm}^{-1}$.

Os espectros eletrônicos foram obtidos em metanol através do espectrofotômetro Agilent 8453 UV-visible Spectroscopy System. Os compostos foram submetidos à varredura de 190 a $1000 \mathrm{~nm}$, usando uma cubeta de quartzo de $1 \mathrm{~cm}$ de caminho óptico. A partir dos máximos de absorção obtidos nos espectros foram determinados os coeficientes de absortividades molares.

\section{Resultados e discussão}

\subsection{Espectroscopia Víbracional na região do infravermelho}

$\mathrm{O}$ espectro de infravermelho do ligante 3-MeOsalen apresentou estiramentos característicos, tais como o $v \mathrm{C}=\mathrm{N}$, localizado em $1632 \mathrm{~cm}^{-1}$, $v \mathrm{C}-\mathrm{O}$ do fenol em $1295 \mathrm{~cm}^{-1}$, o vass $\mathrm{C}-\mathrm{O}-\mathrm{C}$ e o $v_{\mathrm{s}} \mathrm{C}-\mathrm{O}-\mathrm{C}$ em 1245 e $1081 \mathrm{~cm}^{-1}$, respectivamente. Estes estiramentos indicam a presença do grupo imina da base de Schiff e a presença do grupo metoxila da orto-vanilina. Já o espectro de infravermelho do ligante 3-MeOsalophen apresentou os mesmos estiramentos como o $\mathrm{vC}=\mathrm{N}$ em $1611 \mathrm{~cm}^{-1}$, vC-O do fenol em $1256 \mathrm{~cm}^{-1}$, o vass $\mathrm{C}-\mathrm{O}-\mathrm{C}$ e o $v_{\mathrm{s}} \mathrm{C}-\mathrm{O}-\mathrm{C}$ em 1247 e $1076 \mathrm{~cm}^{-1}$, respectivamente.

Como efeitos na formação dos complexos mononucleares [Fe(3-MeOsalen)Cl] e [Fe(3-MeOsalophen) $\mathrm{Cl}$ ] observaram-se deslocamentos para menores números de onda do $\mathrm{v} \mathrm{C}=\mathrm{N}$ e $v \mathrm{C}-\mathrm{O}$. Os estiramentos $v \mathrm{C}=\mathrm{N}$ destes complexos foram deslocados para 1630 e $1600 \mathrm{~cm}^{-1}$ e o vC-O em 1286 e $1253 \mathrm{~cm}^{-1}$ para os [Fe(3-MeOsalen)Cl] e [Fe(3-MeOsalophen)Cl], 
respectivamente. Através dos deslocamentos das bandas no espectro do complexo em relação ao correspondente ligante livre foi possível confirmar a efetividade da reação de complexação.

Nos espectros dos complexos [Fe(3-MeOsalen) $\mathrm{NO}_{2}$ ] observou-se bandas referentes aos estiramentos assimétricos do íon $\mathrm{NO}_{2}$ em $1317 \mathrm{~cm}^{-1}$. Enquanto que os estiramentos simétricos apareceram em $1271 \mathrm{~cm}^{-1}$ e $1270 \mathrm{~cm}^{-1}$ e a banda de deformação angular ONO foi visualizada em $833 \mathrm{~cm}^{-1}$ e $831 \mathrm{~cm}^{-1}$ para os [Fe(3-MeOsalen) $\mathrm{NO}_{2}$ ] e [Fe(3-MeOsalophen) $\mathrm{NO}_{2}$ ], respectivamente. A presença destas bandas no espectro de IV dos complexos indicaram que a coordenação do íon nitrito deve ter ocorrido através do átomo de nitrogênio e de forma monodentada, pois a diferença entre o estiramento assimétrico e simétrico está abaixo de $100 \mathrm{~cm}^{-1}$. A diferença entre $v\left(\mathrm{NO}_{2}\right)_{\text {ass }}$ e $v\left(\mathrm{NO}_{2}\right)_{\text {sim }}$, observadas no espectro foi de $46 \mathrm{~cm}^{-1}$ para 0 [Fe(3-MeOsalen) $\mathrm{NO}_{2}$ ]. O procedimento principal para identificação dos modos de coordenação do íon nitrito por infravermelho, segundo a literatura, é realizada através da avaliação da diferença entre as frequências dos estiramentos $\mathrm{N}-\mathrm{O}$ simétricos e assimétricos. Nos nitro complexos, essas diferenças são menores que $100 \mathrm{~cm}^{-1}$ e nos nitrito complexos são maiores do que $300 \mathrm{~cm}^{-1}$ (NAKAMOTO, 1997). Na figura 3 é apresentada a sobreposição dos espectros de infravermelho dos nitro complexos.

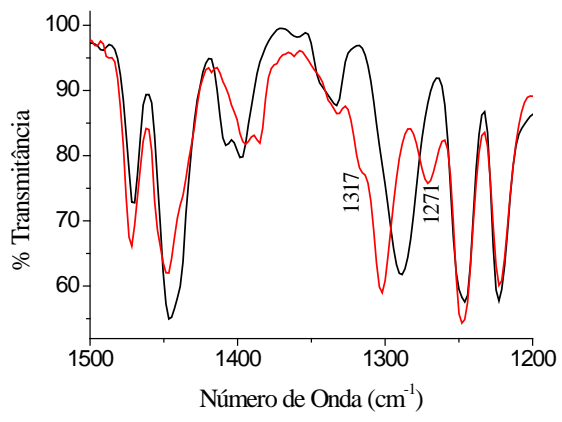

(a)
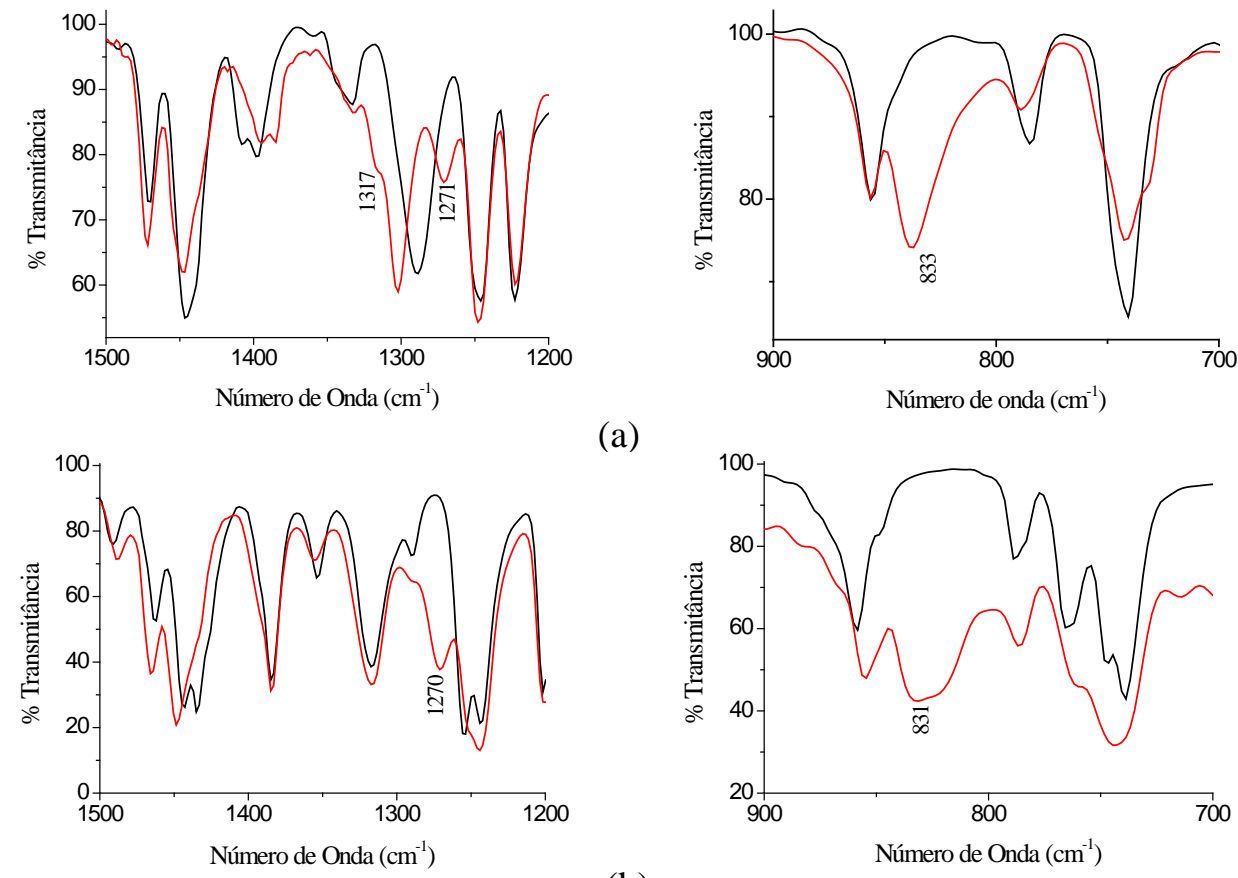

(b)

Figura 3- Sobreposição dos espectros de infravermelho (a) - $[\mathrm{Fe}(3-\mathrm{MeOsalen}) \mathrm{Cl}]$ e - $\left[\mathrm{Fe}(3-\mathrm{MeOsalen}) \mathrm{NO}_{2}\right]$ e (b) —[Fe(3-MeOsalophen)Cl] e - [Fe(3-MeOsalophen) $\left.\mathrm{NO}_{2}\right]$ na região de $1500-1200 \mathrm{~cm}^{-1}$ e de $900-700 \mathrm{~cm}^{-1}$

Na tabela 1 são apresentados os principais modos vibracionais e as respectivas atribuições observados nos espectros de infravermelho do 3-MeOsalen, 3-MeOsalophen e dos complexos de ferro. 
Tabela 1- Atribuições dos principais modos vibracionais presentes ( $v$ - estiramento e $\delta$ - deformação angular) no espectro de infravermelho dos compostos em estudo.

\begin{tabular}{c|c|c|c|c|c|c}
\hline Composto & $v \mathrm{C}=\mathrm{N}$ & $v \mathrm{C}-\mathrm{O}$ & $v\left(\mathrm{NO}_{2}\right)_{\text {ass }}$ & $v\left(\mathrm{NO}_{2}\right)_{\text {sim }}$ & $\delta(\mathrm{ONO})$ & $v \mathrm{Fe}-\mathrm{O}$ \\
\hline 3-MeOsalen & 1632 & 1295 & ---- & ---- & ---- & --- \\
\hline 3-MeOsalophen & 1611 & 1256 & ---- & ---- & ---- & ---- \\
\hline$[\mathrm{Fe}(3-M e O s a l e n) \mathrm{Cl}]$ & 1630 & 1286 & ---- & ---- & ---- & 442 \\
\hline$[\mathrm{Fe}(3-M e O s a l o p h e n) \mathrm{Cl}]$ & 1600 & 1253 & ---- & ---- & ---- & 456 \\
\hline$\left[\mathrm{Fe}(3-M e O s a l e n) \mathrm{NO}_{2}\right]$ & 1617 & 1303 & 1317 & 1271 & 833 & 434 \\
\hline$\left[\mathrm{Fe}(3-M e O s a l o p h e n) \mathrm{NO}_{2}\right]$ & 1607 & 1255 & ---- & 1270 & 831 & 448 \\
\hline
\end{tabular}

(Fonte: Próprio Autor)

\subsection{Espectroscopia de absorção na região do ultravioleta e visível}

Os ligantes pertencentes as classes de compostos orgânicos bases de Schiff apresentam transições eletrônicas entre os níveis de energia $\pi-\pi^{*}$ e $n-\pi^{*}$, referentes ao grupo imino $(C=N)$. Para os derivados de base de Schiff salen, a banda $\pi-\pi^{*}$ foi verificada na região entre $317 \mathrm{e}$ $370 \mathrm{~nm}$ e a $\mathrm{n}-\pi^{*}$ aparece na região entre 385 e $420 \mathrm{~nm}$ (ZAMIAN; DOCKAL, 1996).

O espectro do ligante 3-MeOsalen foi obtido em metanol na concentração de $1.10^{-5} \mathrm{molL}^{-1}$ e observaram-se cinco bandas com comprimentos de onda máximos em 221, 263, 294, 324 e $421 \mathrm{~nm}$. As bandas são atribuídas às transições eletrônicas $\pi-\pi^{*}$, dos grupos cromóforos $\mathrm{C}=\mathrm{C}, \mathrm{C}=\mathrm{N}$ e a transição $\mathrm{n}-\pi^{*}$, pertinente aos pares de elétrons não ligantes. $\mathrm{O}$ espectro do ligante 3-MeOsalophen foi obtido em metanol na concentração de $5.10^{-5} \mathrm{molL}^{-1} \mathrm{e}$ observaram-se cinco bandas com comprimentos de onda máximos em 221, 279, 326 e $454 \mathrm{~nm}$. As transições eletrônicas em 221, 279, $326 \mathrm{~nm}$ são característicos das bandas $\pi$ - $\pi^{*}$ enquanto que a absorção em $454 \mathrm{~nm}$ é referente ao grupo imino. Na figura 4 é apresentada a sobreposição dos espectros eletrônicos dos ligantes.

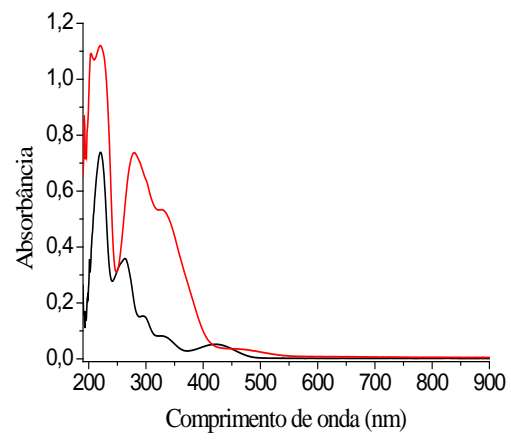

Figura 4 - Sobreposição dos espectros eletrônicos em metanol do -3-MeOsalen e -3-MeOsalophen

Nos complexos de Fe(III) com cada ligante são observados deslocamentos batocrômicos das bandas devido a coordenação do ligante ao íon metálico. No espectro eletrônico do [Fe(3-MeOsalen)Cl], obtido em metanol, são observadas seis bandas com comprimento de onda de 230, 273, 302, 342, 434 e 533 nm. As cinco primeiras absorções são atribuídas às transições intraligantes que se deslocam para maiores comprimentos de onda se comparadas às transições do ligante livre. A banda adicional em $533 \mathrm{~nm}$ refere-se à transição de transferência de carga LMCT, do ligante cloreto $\left(\mathrm{p} \pi \mathrm{Cl}^{-}\right)$para o metal $\left(\mathrm{d} \pi \mathrm{Fe}^{3+}\right)$. Enquanto no complexo [Fe(3-MeOsalophen)Cl] são verificadas transições em 233, 307, 347, 415 e 634 nm. Estas quatro primeiras transições são referentes as bandas intraligantes e a banda em $634 \mathrm{~nm}$ refere-se também a mesma transição de transferência de carga LMCT. 
Ao substituir o ligante cloreto por um ligante de interesse biológico, no caso o íon nitrito, que é um ligante $\pi$ receptor, proporciona alterações no perfil dos espectros eletrônicos dos nitro complexos [Fe(3-MeOsalen) $\left.\mathrm{NO}_{2}\right]$ e $\left[\mathrm{Fe}(3-\mathrm{MeOsalophen}) \mathrm{NO}_{2}\right]$. Para os dois nitro complexos foram averiguados leves deslocamentos nas transições intraligantes e um dos indícios para a formação do complexo foi pela ausência da banda de transferência de carga LMCT dos complexos de partida como é observado na figura 5 através da sobreposição dos complexos de partidas e dos nitro complexos.

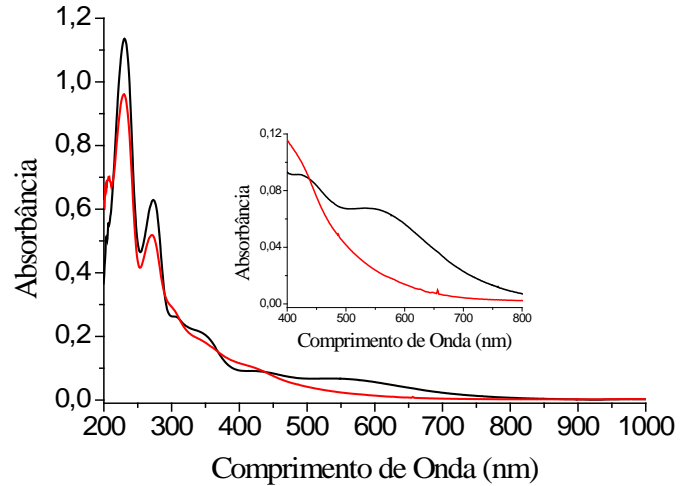

(a)

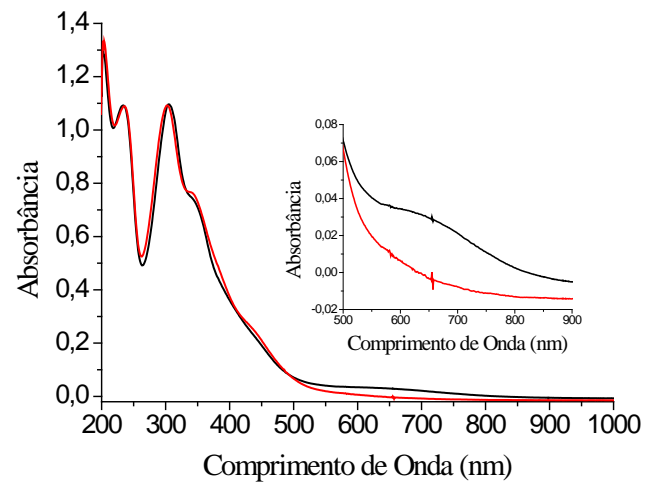

(b)

Figura 5- Sobreposição dos espectros eletrônicos (a) - $[\mathrm{Fe}(3-\mathrm{MeOsalen}) \mathrm{Cl}]$ e — [Fe(3-MeOsalen) $\left.\mathrm{NO}_{2}\right]$ e (b) —[Fe(3-MeOsalophen)Cl] e —[Fe(3-MeOsalophen) $\left.\mathrm{NO}_{2}\right]$

Na tabela 2 são relatados os valores dos comprimentos de onda do ligante 3-MeOsalen, 3-MeOsalophen, [Fe(3-MeOsalen)Cl], [Fe(3-MeOsalophen)Cl], [Fe(3-MeOsalen) $\mathrm{NO}_{2}$ ] e [Fe(3-MeOsalophen) $\mathrm{NO}_{2}$ ] e os valores de absortividades molares.

Tabela 2- Comparativo das bandas (nm) encontradas nos espectros dos compostos em estudo.

\begin{tabular}{c|c|c|c|c|c|c}
\hline Composto & \multicolumn{5}{|c}{ Comprimento de onda, nm (Absortividade molar) } \\
\hline 3-MeOsalen & 221 & 263 & 294 & 324 & 421 & ---- \\
& $\left(7,4.10^{4}\right)$ & $\left(3,6.10^{4}\right)$ & $\left(1,6.10^{4}\right)$ & $\left(8,2.10^{3}\right)$ & $\left(5,2.10^{3}\right)$ & \\
\hline 3-MeOsalophen & 223 & 279 & ---- & 332 & 462 & ---- \\
& $\left(4,5.10^{4}\right)$ & $\left(2,7.10^{4}\right)$ & & $\left(2.10^{4}\right)$ & $(824)$ & \\
\hline$[\mathrm{Fe}(3-M e O s a l e n) \mathrm{Cl}]$ & 230 & 273 & 302 & 342 & 434 & 533 \\
& $\left(2,9.10^{4}\right)$ & $\left(1,6.10^{4}\right)$ & $\left(7,4.10^{3}\right)$ & $\left(5,7.10^{3}\right)$ & $\left(2.5 .10^{3}\right)$ & $\left(2,0.10^{3}\right)$ \\
\hline$[\mathrm{Fe}(3-M e O s a l o p h e n) C l]$ & 233 & 307 & ---- & 347 & 415 & 634 \\
& $\left(4,9.10^{4}\right)$ & $\left(5,1.10^{3}\right)$ & & $\left(1,3.10^{4}\right)$ & $\left(1,2.10^{4}\right)$ & $\left(1,4.10^{3}\right)$ \\
\hline$\left[\mathrm{Fe}(3-M e O s a l e n) \mathrm{NO}_{2}\right]$ & 230 & 271 & 306 & 352 & 428 & ---- \\
& $\left(4,4.10^{4}\right)$ & $\left(2,3.10^{4}\right)$ & $\left(1,2.10^{4}\right)$ & $\left(7,7.10^{3}\right)$ & $\left(4,2.10^{3}\right)$ & \\
\hline$\left[\mathrm{Fe}(3-M e O s a l o p h e n) \mathrm{NO}_{2}\right]$ & 235 & 302 & ---- & 336 & 416 & ---- \\
& $\left(2,9.10^{4}\right)$ & $\left(3,0.10^{4}\right)$ & & $\left(2,1.10^{4}\right)$ & $\left(8,5.10^{3}\right)$ & \\
\hline Atribuição & $\pi-\pi^{*}$ & $\pi-\pi^{*}$ & $\pi-\pi^{*}$ & $\pi-\pi^{*}$ & $\mathrm{n}-\pi^{*}$ & $\mathrm{p} \pi-\mathrm{d} \pi^{*}$ \\
\hline
\end{tabular}

(Fonte: Próprio Autor) 


\section{Conclusões}

Pelas técnicas espectroscópicas empregadas foi possível indicar a formação dos ligantes e dos complexos precursores de ferro com as bases de Schiff 3-MeOsalen e 3-MeOsalophen, assim como dos respectivos complexos contendo o ligante nitro. A partir dos dados de infravermelho, observou-se alterações nos modos vibracionais e estiramentos específicos do grupo imina e nitro que comprovaram a formação de complexos com a base de Schiff e com o ligante nitro. Nos espectros eletrônicos obtidos foram visualizadas variações de energias das transições eletrônicas, presença da banda de transferência de carga do cloreto para o centro metálico e a ausência desta banda nos nitro complexos.

\section{Iron(III) nitro complexes with Schiff base salen derivatives}

Albstract: The iron(III) complexes with Schiff base salen present relevant applications such as chemotherapeutic agents and materials chemistry. Seeking to develop Schiff base coordination compounds with improved pharmacologics properties it was used nitrite ion as ligand due nitro complex usually act as pro-drug releasing nitric oxide in biological environment, an important cell protection agent and vasodilator. In this study were obtained new iron(III) nitro complexes of ligands derivatives from salen Schiff bases, being synthesized ligands 3-MeOsalen and 3-MeOsalophen from ethylenediamine and ortho-phenylenediamine, respectively, and the aldehyde ortho-vanillin. The ligands 3-MeOsalen and 3-MeOsalophen and complexes [Fe(3MeOsalen)Cl], [Fe(3-MeOsalophen)Cl], [Fe(3-MeOsalen)NO $\mathrm{NO}_{2}$ and [Fe(3-MeOsalophen) $\mathrm{NO}_{2}$ ] were synthetized and characterized by vibrational spectroscopy in the infrared region and electronic spectroscopy in the ultraviolet and visible region.

The infrared spectra allowed the characterization of the ligands 3-MeOsalen and 3-MeOsalophen, mostly by the presence of $v \mathrm{C}=\mathrm{N}$ near $1620 \mathrm{~cm}^{-1}$. The synthesis of the precursor complexes as evidenced by changes in the vibrational frequencies of $v \mathrm{C}=\mathrm{N}, v \mathrm{C}-\mathrm{O}$ and the presence of metal-oxygen and metal-nitrogen vibrational modes. The iron nitro complexes exhibited $v\left(\mathrm{NO}_{2}\right)_{\text {ass }}$ around $1300 \mathrm{~cm}^{-1}$ and $v\left(\mathrm{NO}_{2}\right)_{\text {sim }}$ at $1271 \mathrm{~cm}^{-1}$ and angular deformation around $830 \mathrm{~cm}^{-1}$, indicating so that the coordination is done via the nitrogen atom.

Electronic spectra of the Schiff bases were marked by the presence of $\pi-\pi^{*}$ electronic transitions from aromatic ring and imino group and $n-\pi^{*}$ transitions related to free-electron pairs. For the complexes [Fe(3-MeOsalen)Cl] and [Fe(3-MeOsalophen)Cl] was evidenced shifts of intraligands transitions and the emergence of new bands such as LMCT $\left(p \pi \mathrm{Cl}^{-} \rightarrow d \pi^{*} \mathrm{Fe}^{3+}\right)$. Such charge transfer were not observed in the spectra of [Fe(3-MeOsalen) $\mathrm{NO}_{2}$ ] and [ $\mathrm{Fe}\left(3-\mathrm{MeO}\right.$ salophen) $\mathrm{NO}_{2}$ ] indicating so the chloride ligand replacement by nitrite ion.

Keywords: Complex; Schiff base; salen; nitro.

\section{Referências bibliográficas}

ANSARI, Khairul I.; GRANT, James D.; KASIRI, Sahba, WOLDEMARIAM, Getachew A.; SHERESTHA, Bishakha; MANDAL, Subhrangsu S. Manganese (III)-salens induce tumor selective apoptosis in human cells, Journal of Inorganic Biochemistry, 103, 5: 2009.

ARANHA, Pedro E.; SANTOS, Mirian P. dos; ROMERA, Sandra; DOCKAL, Edward R. Synthesis, characterization, and spectroscopic studies of tetradentate Schiff base chromium(III) complexes, Polyhedron, 26, 7: 2007. 
COZZI, Pier Giorgio. Metal-Salen Schiff base complexes in catalysis: practical aspects, Chemical Society Reviews, 33, 7: 2004.

DEHKORDI, Maryam Nejat; LINCOLN, Per. Comprehensive Study on the Binding of Iron Schiff Base Complex with DNA and Determining the Binding Mode, Journal of Fluorescence, 23, 4: 2013.

GARTHWAITE, John. Glutamate, nitric oxide and cell-cell signalling in the nervous system, Trends in Neurosciences, 14, 2: 1991.

GHAFFARI, Abolfazl; BEHZAD, Mahdi; POOYAN, Mahsa; RUDBARI, Hadi Amiri; BRUNO, Giuseppe. Crystal structures and catalytic performance of three new methoxy substituted salen type nickel(II) Schiff base complexes derived from meso-1,2-diphenyl-1,2ethylenediamine, Journal of Molecule Structure, 1063, 2014.

HIBBS JR., John B.;TAINTOR, Read R. VAVRIN, Zdnek; RACHLIN, Elliot M. Nitric oxide: a cytotoxic activated macrophage effector molecule, Biochemical and biophysical research communications, 157, 1: 1988.

IGNARRO, Louis J. Endothelium-derived nitric oxide: actions and properties, The FASEB Journal, 3, 1: 1989.

LEE, Soo-Young; HILLE, Annegret; KITANOVIC, Igor; JESSE, Patrick; HENZEA, Günter; WÖLFL , Stefan; GUST , Ronald ; PROKOP, Aram. [FeIII(salophene)Cl], a potent iron salophene complex overcomes multiple drug resistance in lymphoma and leukemia cells, Leukemia Research, 35, 3: 2011.

MUHAMMAD, Nafees; GUO, Zijian. Metal-based anticâncer chemotherapeutic agents, Current opinion in chemical biology, 19, 2014.

NAKAMOTO, Kazuo. Infrared and raman spectra of inorganic and coordination compounds - Part B. Wiley-Interscience. New York: 1997.

SALOMÃO, Gisele C.; OLSEN, Mara H. N.; DRAGO, Valderes; FERNANDES, Christiane; FILHO; Cardoso L.; ANTUNES, O. A. C.; Oxidation of cyclohexane promoted by [Fe(III)(Salen)Cl] and [Mn(III)(Salen)Cl], Catalysis Communications, 8, 1: 2007.

WINK, David A.; MITCHELL, James B. Serial Review: Nitric Oxide in Cancer Biology and Treatment, Free Radical Biology \& Medicine, 34, 8: 2003.

WOLDEMARIAM, Getachew A.; MANDAL, Subhrangsu S. Iron(III)-salen damages DNA and induces apoptosis in human cell via mitochondrial pathway, Journal of Inorganic Biochemistry, 102, 4: 2008.

ZAHRAN, Z. N.; CHOOBACK, L.; COPELAND, D.M., West, A. N.; RICHTER-ADDO, G. B. Crystal structures of manganese- and cobalt-substituted myoglobin in complex with NO and nitrite reveal unusual ligand conformations, Journal of Inorganic Biochemistry, 102, 2: 2008.

ZAMIAN, José R.; DOCKCAL, Edward. Tetradentate Schiff base oxovanadium(IV) complexes, Transition metal chemistry, 21, 1: 1996. 